\title{
Human Mandible Prenatal Morphogenesis
}

\author{
Sergey Lvovich Kabak ${ }^{1}$ Natalia Victorovna Zhuravleva ${ }^{1} \quad$ Yuliya Michailovna Melnichenko $^{1}$ \\ ${ }^{1}$ Human Morphology Department, Belarusian State Medical \\ University, Minsk, Belarus \\ J Morphol Sci 2019;36:57-62. \\ Address for correspondence Sergey Lvovich Kabak, PhD, Human \\ Morphology Department, Belarusian State Medical University, \\ Dzerzhinsky Avenue 83, 220116, Minsk, Belarus \\ (e-mail: kabakmorph@gmail.com).
}

Abstract
Keywords
- mandible
- human development
- Meckel's cartilage
- chondroid tissue
- condylar cartilage

Introduction This study was performed with the aim of detecting the interaction between cartilaginous and bone tissues in the process of mandible morphogenesis in human embryos.

Material and Methods Whole-mount skeletal preparations, stained with Alcian blue and Alizarin red, as well as serial histological sections impregnated with silver and stained with hematoxylin and eosin were studied. A total of 48 human embryos/fetuses from 5 to 29 weeks of age were used.

Results In the mandible anlage, in addition to the woven bone, cartilaginous and chondroid tissues are present. Cartilaginous tissue islets are localized at the tip of the condylar and coronoid processes and also in the region of the mandibular symphysis. The chondroid tissue is incorporated into the bone in the region of the mandibular symphysis, along the edge of the coronoid process, and also in the alveolar part of the mandible.

Conclusion Meckel's cartilage participates in the formation of the mandibular body, and its remains persist in the area of the mandibular symphysis until the second half of the prenatal development.

\section{Introduction}

In an adult, the mandible is an unpaired bone that forms part of the facial skull. In newborns, it consists of two halves separated by a mandibular symphysis, which, in addition to fibrous connective tissue, includes fragments of cartilaginous tissue (chondriola symphysea), as well as one or more ossicula mentalia. ${ }^{1}$ The two halves of the mandible and ossicula mentalia combine into a single bone in the first year of life (by 9-12 months).

The mandible as the derivative of the first pharyngeal arch develops from the cells of the neural crest, which migrate to the mandibular processes of the mandibular arch in the 4th week of embryonic development. ${ }^{2}$ Differentiation of the cells of the ectomesenchyme of the first pharyngeal arch begins after ingrowth of the maxillary nerve, which, due to neurotropic factors, induces chondrogenesis and osteogenesis.

An important role in the mandible morphogenesis is played by Meckel's cartilage-"the primary lower jaw." It is a template by which the size of the primary ossification centers increases. In the experiment, external influences that disrupt the growth of Meckel's cartilage lead to mandibular hypoplasia. $^{3}$

At present, the question of Meckel's cartilage ossification and its incorporation into the mandibular body remains controversial. In addition, the participation of secondary cartilage in the formation of the mandible has not been adequately described in the literature.

The purpose of this study is to detect an interaction between cartilaginous and bone tissues in the process of mandible morphogenesis in human embryos.

\section{Material and Methods}

Forty-eight human embryos/fetuses between 5 and 29 weeks in utero and without visible developmental anomalies were studied. Their age was determined by the crown-rump length (CRL) or according to obstetric anamnesis ( - Table 1). Part of the material was totally stained with Alcian blue and Alizarin red, followed by clearing in an alkaline solution. Serial histological sections of entire embryos from the collection of the Normal Anatomy Department and Histology, Cytology and received

August 12, 2018

accepted

February 15, 2019
DOI https://doi.org/

10.1055/s-0039-1685456. ISSN 2177-0298.
Copyright $\odot 2019$ by Thieme Revinter

Publicações Ltda, Rio de Janeiro, Brazil
License terms

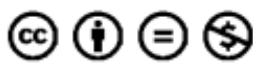


Table 1 Age of embryos/fetuses used in the study

\begin{tabular}{|c|c|c|c|c|c|}
\hline Size (CRL, mm) & Age (days/weeks) & Number of embryos & Size (CRL, mm) & Age (days/weeks) & Number of embryos \\
\hline 5 & 28 days ${ }^{* *}$ & $1 \dagger$ & - & 12 weeks $\diamond$ & $1 \downarrow$ \\
\hline 6 & 28 days $* *$ & $7 \dagger$ & - & 12 weeks $\diamond$ & $2 \ddagger$ \\
\hline 13 & 41 days $^{* *}$ & $6 \dagger$ & - & 13 weeks $\diamond$ & $1 \bullet$ \\
\hline 15 & 44 days** & $1 \dagger$ & - & 13 weeks $\diamond$ & $4 \ddagger$ \\
\hline 17 & 47 days ${ }^{* *}$ & $4 \dagger$ & 73 & $13+3$ weeks $^{\wedge}$ & $1 \dagger$ \\
\hline 19 & 49 days $^{* *}$ & $1 \dagger$ & - & 14 weeks $\diamond$ & $1 \ddagger$ \\
\hline 27 & 54 days $* *$ & $1 \dagger$ & - & 16 weeks $\diamond$ & $1 \ddagger$ \\
\hline- & 10 weeks $^{\wedge}$ & $1 \bullet$ & - & 20 weeks $\diamond$ & $5 \ddagger$ \\
\hline- & 11 weeks $\diamond$ & 4 & - & 21 weeks $\diamond$ & $1 \ddagger$ \\
\hline 48 & $11+3$ weeks $^{\wedge}$ & $4 \dagger$ & - & 29 weeks $\diamond$ & $1 \ddagger$ \\
\hline
\end{tabular}

Abbreviation: CRL, crown-to-rump length.

Notes:

** www.ehd.org/developmental-stages/stage0.php.

^http://onlinelibrary.wiley.com/doi/10.1002/uog.13448/full.

$\diamond$ gestational age (according to obstetric anamnesis).

$\dagger$ - serial histological sections of the embryos.

$\ddagger$ - histological sections of the mandible fragments.

$\checkmark$ - whole-mount skeletal preparations.

Embryology Department of the Belarusian State Medical University together with individual fragments of the mandibleimpregnated by silver nitrate, according to Bilshovsky-Buke, and stained with hematoxylin and eosin-were also studied. The present study was approved by the Medical Ethics Committee of the Belarusian State Medical University, Minsk, Belarus (Protocol No.: 2017/2).

The present study was approved by the Medical Ethics Committee of the Belarusian State Medical University, Minsk, Belarus (Protocol No.: 2017/2). Photos of anatomical and histological specimens were obtained using a Leica Microsystems stereomicroscope (Leica Microsystems, GmbH, Wetzlar, Germany) and an optical microscope Leica DM2500 (Leica Microsystems).

\section{Results}

By the beginning of the 5th week of embryogenesis, the lower wall of the stomodeum is formed from the fusion of the two mandibular processes. In human embryos with a CRL of $13 \mathrm{~mm}$, in the mandibular processes, next to the third branch of the trigeminal nerve, a Meckel's cartilage anlage in the form of mesenchyme condensation is revealed. In the 7th week of prenatal development, Meckel's cartilage is a paired typical hyaline cartilage surrounded by perichondrium that extends from the ear capsule to the midline, where the cartilages of opposite sides are separated by a layer of mesenchymal tissue. On the lateral side of the Meckel's cartilage, there is the inferior alveolar nerve, on the medial side-the lingual nerve, which is directed toward the tongue anlage.

In embryos with a CRL of $13 \mathrm{~mm}$ (7 weeks), on the histological sections from both sides of the midline outside the Meckel's cartilage, the primary center of ossification appears in the thickness of the mesenchyme of the mandib- ular processes (-Fig. 1A). In this area, the bony trabeculae containing the oxyphilic extracellular matrix are formed. Presence of bony trabeculae indicates the beginning of organic matrix (osteoid) secretion by osteoblasts. The primary center of ossification first looks like a plate located on the lateral side of the Meckel's cartilage and is separated from it by the inferior alveolar nerve. In the following, the process of intramembranous ossification extends downward, backward, forward and upward. By the end of the embryonic period, the anlage of the mandibular body is formed by two bone plates. They partially merge, resulting in the formation of the Y-shaped structure, which, in the form of a groove, covers the inferior alveolar nerve from below (-Fig. 1B). The medial bone plate is in close contact with the perichondrium of Meckel's cartilage. There is a wide gap (the area of the future mental foramen) in the lateral bone plate. At this point, the inferior alveolar nerve turns to the side and appears outside the lateral bone plate continuing as the mental nerve (-Fig. 1C, D).

In the serial sections of the embryo with a CRL of $48 \mathrm{~mm}$ (12th week of development), mesenchymal condensation is revealed in the region of the future condylar process. It is located on the distal (posterior) end of the bony trabeculae of the mandibular body, covered with the periosteum. Soon the mesenchymal condensation is replaced by a cartilaginous tissue. Due to its rapid increase in volume in fetuses at 11 to 12 weeks of development, cartilage (the core of the condylar process) is formed, which looks like an inverted cone (-Fig. 1E). Its broad base is turned toward the mandibular fossa of the temporal bone, and the apex is directed in the opposite direction, reaching the level of the mandibular foramen. Outside the cartilage, a periosteal bone collar is formed. The collar causes dystrophic changes in the underlying cartilage that manifest hypertrophy of the 


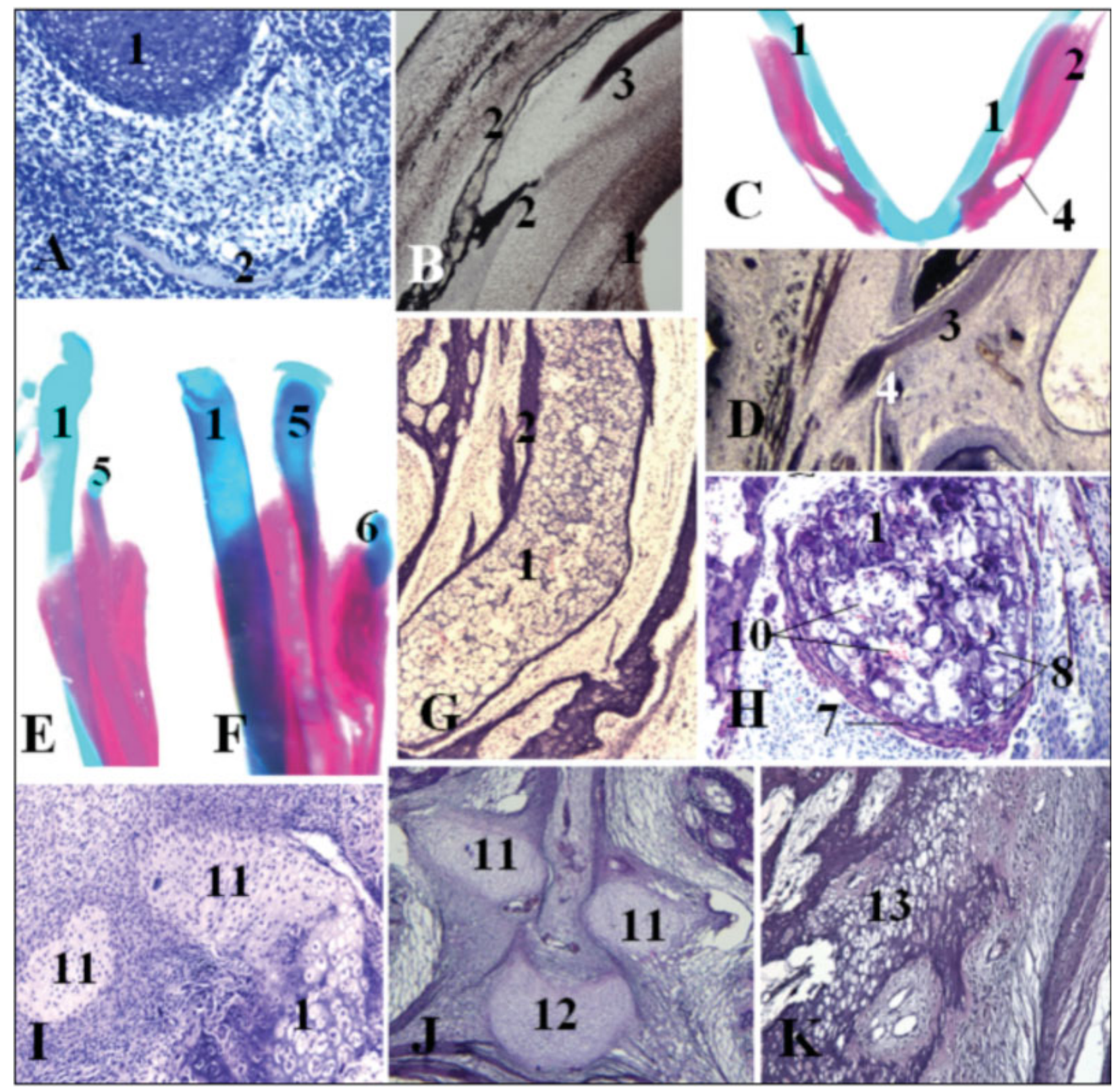

Fig. 1 Organogenesis and histogenesis of the mandible in human embryos ( $\mathrm{A}-13 \mathrm{~mm}$ CRL, B - $27 \mathrm{~mm}$ CRL, C - 10 weeks, D - 73 mm CRL, E - 11 weeks, F-12 weeks, G, H- 13 weeks, I - 16 weeks; J, K - 20 weeks). 1-Meckel's cartilage, 2-bone plates, 3-inferior alveolar nerve, 4-mental foramen, 5-condylar cartilage, 6-coronoid cartilage, 7-periosteal bone collar, 8-hypertrophic chondrocytes, 9-calcified cartilage matrix, 10-mesenchyme with blood vessels; 11-Meckel's cartilage remnants, 12-secondary cartilage, 13-chondroid tissue in the mandibular symphysis. A, B, D, G-K - photomicrographs of histological preparations; stain: A, H, I-K - hematoxylin-eosin; B, D, G - impregnation with silver nitrate according to Bilshovsky-Buke, magnification: A, H - x200; B, D, G, I - x100; J, K - x40; C, E, F - total preparations; stain: Alcian blue and Alizarin red.

chondrocytes, the vacuolization of their cytoplasm and karyopycnosis, and the mineralization of the matrix in the form of deep purple spicules. Shortly after the appearance of condylar cartilage, the periosteal collar leaves only a small portion of it uncovered at the very end of the condylar process-the head of the mandible.

In the human fetus with a CRL of $73 \mathrm{~mm}$ (14th week of development), the head of the mandible and mandibular fossa form temporomandibular joint. An articular cleft completely separates the surfaces of the bony anlages from the articular disc. In human fetuses, starting at 12 weeks of age, the cartilage of the head gradually differentiates into several layers. Twenty-week-old fetuses have superficially located elongated cells-the perichondrium (fibroblastic layer), which extends into the periosteum of the mandibular ramus. A layer of polymorphic cells surrounded by a small amount of basophilic intercellular matrix lies deeper continuing in the wide zone of flat cells. The layer of hypertrophic chondrocytes borders on the zone of cartilage erosion. From the side of the mesenchyme surrounding the cartilage, vascular channels grow into the mandibular condyle. At 12 to 16 weeks of intrauterine development, the end of the 
coronoid process of the mandible also reveals a site of cartilage, characterized by alzianophilia on cleared human embryos (-Fig. 1F). Histological examination of 20-weekold fetuses revealed the presence of groups of hypertrophic chondrocytes in partially mineralized matrix, surrounded by areas of woven bone, near which the cartilage is partially destroyed. In addition, a strip of cartilaginous tissue was found along the edge of the coronoid process. Within the oxyphilic bone matrix, simultaneously with osteocytes, populations of densely lying large cells located inside the lacunae are determined. The pericellular region around these cells is characterized by a weak basophilia.

Simultaneously with the appearance of cartilage in the condylar and coronary processes, the transformation of the microscopical structure of the Meckel's cartilage begins. In fetuses in the 11th week of intrauterine development, this structure remains intact from the middle ear to the mandibular symphysis, but somewhat changes the direction of its course. Closer to the distal end, the cartilage sharply deflects laterally and closely interacts with the mandibular body anlage. At this point, the cartilage is surrounded by bone trabeculae on three sides. Only its medial surface remains uncovered by bone. The distal end of the Meckel's cartilage is curved in the medial direction toward the cartilage of the opposite side, from which it is separated by a thin interlayer of mesenchyme. In embryos between 13 and 16 weeks, from the lateral side of the Meckel's cartilage through its direct contact with the bone plate of the jaw anlage, hypertrophic chondrocytes are revealed, the integrity of the cellular lacunae walls are broken, and the intercellular matrix is degraded. The described morphological features are the initial manifestations of the destructive degeneration, which gradually spreads across the cartilage (-Fig. 1G, H). As a result of the destruction of the Meckel's cartilage, cavities filled with an immature myeloid tissue appear, and areas of the calcified extracellular matrix and woven bone from the side of the perichondrium are identified on non-decalcified preparations. The most distal end of the Meckel's cartilage is separated as an independent structure located in the thickness of the mesenchyme separating the two halves of the mandibular body ( - Fig. 1I).

In 16-week-old fetuses, in the region of the mandibular symphysis, next to the remains of the Meckel's cartilage, additional fragments of cartilaginous tissue of round or oval shape-consisting of large chondrocytes in the center and smaller cells along the periphery-are identified.

In fetuses between 20 and 29 weeks in utero, the mandibular body looks open from the part above the groove, which contains teeth and the inferior alveolar nerve and vessels. In the mesenchyme of the caudal portion of the mandibular symphysis, from its lingual side, several areas of the cartilaginous tissue are identified alongside the midline. At this point, the symphysis has the form of a triangular extension with the base facing its lingual side.

The paired cartilaginous structures located on both sides of the midline are considered to be the distal portions of the Meckel's cartilage, which undergo progressive atrophy. Microscopically, these structures look like a part of the Meckel's cartilage, located from the medial side of the body of the mandible closer to its angle. In addition to the remnants of the Meckel's cartilage, there is another unpaired cartilaginous rounded structure in the region of the mandibular symphysis, which is located along the midline (-Fig. 1]). According to the histological structure, this structure is a typical hyaline cartilage.

In the woven bone, at the distal end of the bone anlage of each half of the mandible facing the mandibular symphysis, areas of the cartilaginous tissue are identified, comprising large, densely located cells of oval shape surrounded by a small amount of a homogeneous matrix, which is slightly basophilic in the pericellular region (-Fig. 1K). The presence of a cartilaginous tissue was also found in the alveolar ridge on the lingual side of the mandibular body anlage.

\section{Discussion}

Embryogenesis of the mandible is at the top of the discussions among experts studying the morphogenesis of the maxillofacial region.

We have established that two primary ossification centers fused in the midline of the mandibular processes of the human embryos from outside the Meckel's cartilage, near the inferior alveolar nerve. The time and the place of their appearance are consistent with published data. ${ }^{4,5}$ It indirectly confirms the inductive effect of the nerves and Meckel's cartilage on the appearance of the primary bone in the mesenchyme of the mandibular processes.

Meckel's cartilage is a transient embryonic structure. There are contradictory reports in the literature about the transformation in embryogenesis of its distal fragment, located from the medial side of the bone anlage of the mandible body. Amano et al (2010), ${ }^{6}$ Bontemps et al $(2001)^{7}$ and Lee et al $(2001)^{4}$ believe that the Meckel's cartilage does not undergo endochondral ossification in humans, but atrophies and gradually disappears. According to Radlanski et al $(2016)^{8}$ a site of cartilage, at least along the length from the mental foramen to the midline (at the level of the canine and incisors), is incorporated into the mandible by endochondral ossification. In 14 to 16 week-old fetuses, we observed thinning and subsequent fragmentation of the middle segment of Meckel's cartilage, surrounded by bone tissue from three sides. Chondrocytes, which are the part of this segment, had varying degrees of destructive degeneration, similar to those in endochondral ossification. According to Yang et al. $(2012)^{9}$ the destruction of Meckel's cartilage begins with the accumulation of autophagic vacuoles in the cytoplasm of chondrocytes with products of destruction of the extracellular matrix, which subsequently leads to cell death. Classical apoptosis of the cartilaginous cells takes place during endochondral ossification. Authentic endochondral ossification is characterized by the proliferation of chondrocytes first, and then by their hypertrophy followed by calcification of the extracellular matrix. We observed the calcification of the Meckel's cartilage matrix and the formation of woven bone from the side of the perichondrium on nondecalcified preparations. Thus, we should agree with the opinion of the authors who assert that Meckel's cartilage takes part in the formation of the mandibular body. 
The proximal extremity of Meckel's cartilage also undergoes ossification to form two of the auditory ossicles, the malleus and incus. In mammals the greater part of this embryonic cartilage between the proximal and distal ossified extremities degenerates to become a fibrous tissue in which amorphous remnants of cartilage may persist for some time. In man ossification of all or part of this fibrous tissue along the medial surface of the mandible results in the bony anomaly called mylohyoid bridge. ${ }^{10}$ The ossified middle segment of Meckel's cartilage was present in the symmetrodont Zhangheotherium from Liaoning. ${ }^{11,12}$ This fact supports the assumption that chondrocytes from all parts of Meckel's cartilage can transform into the bone. Yang et al $(2014)^{13}$ show that hypertrophic chondrocytes can survive the cartilage-to-bone transition and become osteoblasts and osteocytes during endochondral bone formation and in bone repair.

Most of the mandible in embryogenesis is formed by direct osteogenesis, although some of its parts develop on basis of secondary cartilage by indirect osteogenesis. ${ }^{8}$ Secondary cartilages appear in embryogenesis later than the primary cartilages forming the chondroskeleton. The periosteum of the primary ossification centers is the source of their development. In the literature, the presence of secondary cartilages was found in the condylar and coronary process, in the mandibular symphysis and the angular region of the mandible, and also in the alveolar ridge at the level of i1, i2, m1, m2, and M1 tooth germs. ${ }^{8,14,15}$ Our data about secondary cartilage origin time in embryogenesis are coincident with published data. ${ }^{8}$

There is a typical hyaline cartilage at the end of the condylar and coronary processes of the mandibular angle, as well as between its two halves in the mandibular symphysis region. We have identified the areas of cartilaginous tissue incorporated into the bone along the edge of the coronoid process, in the alveolar crest and in the region of the mandibular symphysis. It can be classified as cartilaginous (chondroid) tissue ${ }^{1,16}$ considering its location and external resemblance to the cartilaginous and bone tissue simultaneously.

In 20 to 29 week-old-fetuses there are round shape fragments of cartilage-chondriola symphysea-between the mesial ends of the two halves of the mandible body. There are different opinions about the origin and subsequent modification of these structures in the process of embryogenesis in the literature.

Some authors ${ }^{2}$ believe that the chondriola symphysea are the remains of the Meckel's cartilage (Meckelian islets), which turn into the mental ossicula (ossicula mentalia) during ossification. Other researchers hold the view that these cartilages are secondary cartilage, which are formed in the mesenchyme of the mandibular symphysis de novo, ${ }^{17}$ and the distal segment of the Meckel's cartilage is a transient structure. ${ }^{1}$ It has no physiological significance and by the 6th month of intrauterine development disappears as a result of an atrophic degeneration process in the cartilage. ${ }^{7}$ We found the presence of both the primary (Meckel's) and secondary cartilages in the mandibular symphysis region of 16 to 20 week-old fetuses. At the same time, the morphological signs of Meckel's cartilage physiological atrophy, such as a decrease in its size, vacuolization and karyopycnosis of a part of the chondrocytes, the desolation and reduction of the area of the perichondrocytic lacunae, are revealed.

Condylar cartilage appears between the 11th and 12th week of intrauterine development and forms a cartilaginous core of the condylar process, which gradually "plunges" into the bone tissue. In the following, as a result of ossification, most of the cartilage integrates into the condylar process, undergoing endochondral ossification. Finally, cartilaginous tissue remains only in the head of the mandible. The condylar cartilage is an analogue of the epiphyseal cartilage. Proliferation of its cells provides an increase in the size of the mandible both in prenatal and postnatal ontogenesis. ${ }^{14}$ Unlike the epiphyseal plate of long bones, the replacement of condyle cartilage by bone occurs only from the side facing the mandibular body.

The cartilage on the apex of coronoid process appears a bit later than the condyle cartilage. It is a transient structure, which is subsequently replaced by bone.

The transcription factor Sox9 and external mechanical effects such as pressure, ${ }^{8}$ for example, are stimuli that affect the development of cartilage. The secondary cartilage appearance in the mandibular symphysis region in embryogenesis coincides with the first swallowing movements of the fetus. The movements in the temporomandibular joint are also likely to promote the differentiation of the mesenchymal cells of the mandibular condyle into the chondrocytes, as demonstrated in a study by Habib et al (2005). ${ }^{18}$

\section{Conclusion}

In humans, two centers of intramembranous ossification in the mandibular arch appear at the 7th week of embryogenesis. There is a cartilaginous and chondroid tissue in the mandible anlage in addition to the woven bone. Hyaline cartilage is localized at the end of the condylar and coronoid processes, and also as an independent structure in the mandibular symphysis region. The chondroid tissue is incorporated into the bone near the mandibular symphysis and along the edge of the coronoid process. It is also present in the alveolar part of the mandible. Meckel's cartilage is the "primary lower jaw" and persists in the development process until the morphogenesis of the temporomandibular joint is completed and the two halves of the mandible come closer along the midline. In the following, the cartilage undergoes involution and loses its integrity. By the 20 to 29th week of embryogenesis, its remnants persist near the distal part of the body of the mandible and in the mandibular symphysis region.

\section{Funding}

The present study had no sponsorship.

Conflicts of Interest

The authors declare that there are no conflicts of interest. 
62 Human Mandible Prenatal Morphogenesis Kabak et al.

\section{Acknowledgments}

None.

\section{References}

1 Dhem A. The Mandible - Natural History of a Bone Completely Different from All the Others. Dental and Medical Problems 2004; 41(03):395-402

2 Collins P. 2015Embryogenesis. In: Standring S, ed. Gray's Anatomy E-Book: The Anatomical Basis of Clinical Practice. Philadelphia: Elsevier Limited; 32-226

3 Jaskoll T, Abichaker G, Sedghizadeh PP, Bringas P Jr, Melnick M. Cytomegalovirus induces abnormal chondrogenesis and osteogenesis during embryonic mandibular development. BMC Dev Biol 2008;8(08):33

4 Lee SK, Kim YS, Oh HS, Yang KH, Kim EC, Chi JG. Prenatal development of the human mandible. Anat Rec 2001;263(03):314-325

5 Wyganowska-Świątkowska M, Przystańska A. The Meckel's cartilage in human embryonic and early fetal periods. Anat Sci Int 2011;86(02):98-107

6 Amano O, Doi T, Yamada T, et al. Meckel's cartilage: discovery, embryology and evolution: overview of the specificity of Meckel's cartilage. Journal of Oral Biosciences 2010;52(02):125-135

7 Bontemps C, Cannistrà C, Hannecke V, Michel P, Fonzi L, Barbet JP. [The first appearance of Meckel's cartilage in the fetus]. Bull Group Int Rech Sci Stomatol Odontol 2001;43(03):94-99

8 Radlanski RJ, Renz H, Zimmermann CA, Schuster FP, Voigt A, Heikinheimo K. Chondral ossification centers next to dental primordia in the human mandible: A study of the prenatal development ranging between 68 to 270mm CRL. Ann Anat 2016;208:49-57
9 Yang RT, Zhang C, Liu Y, Zhou HH, Li ZB. Autophagy prior to chondrocyte cell death during the degeneration of Meckel's cartilage. Anat Rec (Hoboken) 2012;295(05):734-741

10 Hu Y, Wang Y, Luo Z, Li C. A new symmetrodont mammal from China and its implications for mammalian evolution. Nature 1997;390(6656):137-142

11 Meng JIN, Hu Y, Wang Y, Li C. The ossified Meckel's cartilage and internal groove in Mesozoic mammalian forms: implications to origin of the definitive mammalian middle ear. Zool J Linn Soc 2003;138(04):431-448

12 Ossenberg NS. The mylohyoid bridge: an anomalous derivative of Meckel's cartilage. J Dent Res 1974;53(01):77-82

13 Yang L, Tsang KY, Tang HC, Chan D, Cheah KS. Hypertrophic chondrocytes can become osteoblasts and osteocytes in endochondral bone formation. Proc Natl Acad Sci U S A 2014;111(33): 12097-12102

14 Leander D. Role of Growth Factors in the Development of Mandible. J Indian Orthod Soc 2011;45(02):51-60

15 Mérida Velasco JR, Rodríguez Vázquez JF, De la Cuadra Blanco C, Campos López R, Sánchez M, Mérida Velasco JA. Development of the mandibular condylar cartilage in human specimens of 10-15 weeks' gestation. J Anat 2009;214(01):56-64

16 Bailleul AM, Nyssen-Behets C, Lengelec B, Hall BK, Horner JR. Chondroid bone in dinosaur embryos and nestlings (Ornithischia: Hadrosauridae): Insights into the growth of the skull and the evolution of skeletal tissues. C R Palevol 2016;15(1-2):49-64

17 El Fouhil AF. 2012Development of the site of articulation between the two human hemimandibles (Symphysis Menti). In: Dr. Ken-Ichi Sato (ed). Embryogenesis, InTech 167-188

18 Habib H, Hatta T, Udagawa J, Zhang L, Yoshimura Y, Otani H. Fetal jaw movement affects condylar cartilage development. J Dent Res 2005;84(05):474-479 